\title{
Electron-impact ionization of $\mathrm{Fe}^{8+}$
}

\author{
Aušra Kynienė, Sigitas Kučas, Šarūnas Masys, and Valdas Jonauskas
}

\begin{abstract}
Institute of Theoretical Physics and Astronomy, Vilnius University, Sauletekio av. 3, 10257 Vilnius, Lithuania e-mail: ausra.kyniene@tfai.vu.lt
\end{abstract}

Received 3 July 2018 / Accepted 29 January 2019

\begin{abstract}
Electron-impact ionization cross sections and Maxwellian rate coefficients are presented for the $\mathrm{Fe}^{8+}$ ion by considering processes from the ground and metastable levels. The lifetimes of the levels for the $3 \mathrm{~s}^{2} 3 \mathrm{p}^{5} 3 \mathrm{~d}$ configuration were analysed using the extended basis of interacting configurations. Convergence of the cross sections for the indirect process due to excitations to the high- $n l$ subshells was investigated. We demonstrate that excitations to the subshells with orbital quantum number $l=3$ with subsequent autoionization dominate up to electron energies of $\sim 700 \mathrm{eV}$ for the ground and metastable levels. Modelling of theoretical cross sections obtained for the ground and metastable levels to produce the best fit to the measurements shows that $15 \%$ of ions reach the reaction zone in the metastable state. The obtained results contradict the previous work that showed $\sim 30 \%$ for the metastable fraction.
\end{abstract}

Key words. atomic processes - atomic data

\section{Introduction}

Spectroscopic data provided by the Chandra, XMM-Newton, Solar and Heliospheric Observatory (SOHO), and Hinode satellites opened new opportunities to study astrophysical objects. Iron ions play an important role in the diagnostics of stellar coronae since the corresponding spectra are rich in strong lines (Gabriel et al. 1966; Phillips et al. 2012; Beiersdorfer et al. 2014) and it is an abundant element in the Universe. The Iron project was initiated to compute electron-impact excitation cross sections and rates as well as radiative transition probabilities and photoionization cross sections for the iron ions important in astrophysical applications (Hummer et al. 1993). However, charge state distribution of plasma has to be known since the emission line intensities depend on the ion concentration. Electron-impact ionization and electron-ion recombination define charge state distribution in the collisionally ionized plasmas.

First measurements of the electron-impact ionization cross sections for iron were started by analysing the $\mathrm{Fe}^{+}$ions (Montague et al. 1984). The crossed beams technique was used in the study. Later, this approach was applied to analyse the $\mathrm{Fe}^{2+}$ ion (Mueller et al. 1985). The Lotz formula (Lotz 1968) and the distorted wave (DW) calculations produced good agreement with experimental data. Excitation followed by autoionization (EA) with radiative damping in addition to the direct ionization (DI) were calculated for $\mathrm{Fe}^{23+}$ by Butler \& Moores (1985). They demonstrated that the radiative decay of the excited autoionizing states diminishes the EA cross sections by approximately $20 \%$. In the same way, the $\mathrm{Fe}^{5+}, \mathrm{Fe}^{6+}$, and $\mathrm{Fe}^{7+}$ were analysed by Gregory et al. (1986). The cross sections for $\mathrm{Fe}^{5+}, \mathrm{Fe}^{6+}, \mathrm{Fe}^{9+}$, $\mathrm{Fe}^{11+}$, and $\mathrm{Fe}^{13+}$ were calculated by applying the configurationaverage DW (CADW) method by Pindzola et al. (1986). They provided values for the DI and EA processes that explained previous disagreements with measurements (Gregory et al. 1986). The calculations for the electron-impact ionization cross sections and plasma rate coefficients were extended for the iron isonu- clear sequence (Pindzola et al. 1987). The experimental atomic data of the ionization for $\mathrm{Fe}^{11+}, \mathrm{Fe}^{13+}$, and $\mathrm{Fe}^{15+}$ ions were also reported (Gregory et al. 1987). Atomic iron was experimentally analysed using the crossed-electron-beam - fast-atom-beam method where charge-transfer neutralization was employed to prepare neutral atoms (Freund et al. 1990). The cross section for the lithium-like iron was measured at $4.66 \mathrm{keV}$ using an electron-beam ion trap (Wong et al. 1993). Ion storage ring measurements for the $\mathrm{Fe}^{15+}$ ion (Linkemann et al. 1995) demonstrated the higher quality data compared to the previous crossed beams technique. Later, the electron-impact single ionization cross sections for $\mathrm{Fe}^{q+}(q=1-6,9,10)$ were measured by employing the animated crossed beams technique (Stenke et al. 1999). The DI and EA cross sections using the flexible atomic code (FAC; $\mathrm{Gu}$ 2008) were calculated for all iron ions (Dere 2007). Also, the FAC was used to calculate the electronimpact ionization cross sections for $\mathrm{Fe}^{11+}$ (Kwon \& Savin 2012). Authors managed to improve previous results for this ion (Dere 2007). The Heidelberg heavy-ion storage test storage ring (TSR) was employed to obtain experimental cross sections for $\mathrm{Fe}^{11+}$ (Hahn et al. 2011). The approach used allowed for a significant reduction in the fraction of metastable ions in the beam. Therefore, the measurements were extended to other iron ions, such as $\mathrm{Fe}^{12+}$ (Hahn et al. 2011), $\mathrm{Fe}^{9+}, \mathrm{Fe}^{10+}$ (Hahn et al. 2012), $\mathrm{Fe}^{13+}$ $\mathrm{Fe}^{16+}, \mathrm{Fe}^{17+}$ (Hahn et al. 2013), $\mathrm{Fe}^{14+}$ (Bernhardt et al. 2014), $\mathrm{Fe}^{7+}$ (Hahn et al. 2015), and $\mathrm{Fe}^{8+}$ (Hahn et al. 2016). Recently, the cross sections for $\mathrm{Fe}^{1+}, \mathrm{Fe}^{2+}, \mathrm{Fe}^{3+}$, and $\mathrm{Fe}^{4+}$ ions were produced using the CADW method (Pindzola \& Loch 2018).

It should be noted that experimental cross sections of electron-impact ionization process for the $\mathrm{Fe}^{8+}$ ion were only recently obtained using the heavy-ion storage TSR (Hahn et al. 2016) since previous measurements were not able to deal with large fractions of metastable ions in the beam. Detailed analysis of populations of the levels over time using the Boltzmann distribution for the initial population demonstrated that the $3 s^{2} 3 p^{5} 3 d^{3} F_{4}$ level has the largest population, which amounts to about $57 \%$ during the data collection period (Hahn et al. 2016). 
However, fitting of theoretical data to the experimental cross sections provided value of $\sim 30 \%$ for the metastable fraction. The obtained disagreement suggests that the electron-impact ionization process for $\mathrm{Fe}^{8+}$ has to be investigated using other approaches.

The aim of the current paper is a study of electron-impact single ionization of the $\mathrm{Fe}^{8+}$ ion by performing level-to-level DW (LLDW) calculations. The previous CADW calculations for the ground level of $\mathrm{Fe}^{8+}$ considered DI from all subshells with $n=3$ and EA for the $3 \mathrm{~s} \rightarrow n l(n \leqslant 10)$ excitations (Hahn et al. 2016). For the metastable level, the $3 \mathrm{~s} \rightarrow 4 l$ transitions contributing to the EA process were used from the previous work (Pindzola et al. 1987). On the other hand, our study takes into account the excitations from the $2 s, 2 \mathrm{p}, 3 \mathrm{~s}$, and $3 \mathrm{p}$ subshells for the metastable level. The convergence of the EA cross sections is ensured by considering excitations up to subshells with the principal quantum number $n=20$ and orbital quantum number $l=5$. The obtained data for the ground and metastable levels are fitted to experimental values to determine the fraction of the metastable ions in the beam.

The rest of the paper is organized as follows. Section 2 presents a brief outline of the theoretical approach. In Sect. 3, the obtained results are discussed. Finally, we end with the conclusions from the present investigation.

\section{Theoretical approach}

The FAC ( Gu 2008) is employed to generate energy levels, radiative and Auger transition probabilities, electron-impact excitation, and ionization cross sections for the $\mathrm{Fe}^{8+}$ ion. The code implements the Dirac-Fock-Slater approach. Single configuration approximation is used in the study of ionization cross sections. Therefore, the mixing of configuration state functions corresponding to the same configuration is taken into account. The local central potentials for the ground configurations of every ion are used to generate the necessary bound and continuum wavefunctions for each ion.

Ionization cross sections are studied for the ground and excited $3 \mathrm{~s}^{2} 3 \mathrm{p}^{5} 3 \mathrm{~d}^{3} \mathrm{~F}_{4}$ levels of the $\mathrm{Fe}^{8+}$ ion. The contribution from the excited level to the total electron-impact ionization cross sections is determined by fitting to experimental values. In addition, the change of the population levels over time is investigated by taking the initial population from the Boltzmann distribution for temperature $k_{\mathrm{B}} T_{\mathrm{e}}=87 \mathrm{eV}$ (Hahn et al. 2016). The relative population $p_{i}$ for the level $i$ is determined from the equation

$\frac{\mathrm{d} p_{i}}{\mathrm{~d} t}=\sum_{j>i} p_{j} A_{j i}^{\mathrm{r}}-\sum_{k<i} p_{i} A_{i k}^{\mathrm{r}}$,

where $A_{j i}^{\mathrm{r}}$ is the radiative decay from the level $j$ to the level $i$. The first term on the right hand side defines transitions to the level $i$ from the higher levels while the second term corresponds to the decay from the level $i$ to the lower lying ones. Electric dipole and quadrupole as well as magnetic dipole transitions are calculated for all levels. Electric octupole and magnetic quadrupole transitions are included only for the lowest configurations: $3 \mathrm{~s}^{2} 3 \mathrm{p}^{6}$, $3 s^{2} 3 p^{5} 3 d, 3 s^{2} 3 p^{5} 4 s, 3 s 3 p^{6} 3 d, 3 s^{2} 3 p^{4} 3 d^{2}$.

The radiative transition probabilities from the excited levels have to be known since radiative decays define which levels can contribute to experimental ionization cross sections. The lifetimes of the levels corresponding to the $3 \mathrm{~s}^{2} 3 \mathrm{p}^{5} 3 \mathrm{~d}$ configuration are analysed using the configuration interaction (CI) method. The bases of admixed configurations are generated by employing the configuration interaction strength (CIS; Kučas et al. 1997; Jonauskas et al. 2008, 2011; Karazija \& Kučas 2013):

$$
T\left(K, K^{\prime}\right)=\frac{\sum_{\gamma \gamma^{\prime}}\left\langle\Phi(K \gamma)|H| \Phi\left(K^{\prime} \gamma^{\prime}\right)\right\rangle^{2}}{\bar{E}\left(K, K^{\prime}\right)^{2}} .
$$

Here, $g(K)$ is the statistical weight of the studied configuration $K$ $\left(3 \mathrm{~s}^{2} 3 \mathrm{p}^{5} 3 \mathrm{~d}\right)$ and $\bar{E}\left(K, K^{\prime}\right)$ is the average energy distance between the energy levels of the configurations $K$ and $K^{\prime}$,

$$
\begin{aligned}
\bar{E}\left(K, K^{\prime}\right)= & \frac{\sum_{\gamma \gamma^{\prime}}\left[\langle\Phi(K \gamma)|H| \Phi(K \gamma)\rangle-\left\langle\Phi\left(K^{\prime} \gamma^{\prime}\right)|H| \Phi\left(K^{\prime} \gamma^{\prime}\right)\right\rangle\right]}{\sum_{\gamma \gamma^{\prime}}\left\langle\Phi(K \gamma)|H| \Phi\left(K^{\prime} \gamma^{\prime}\right)\right\rangle^{2}} \\
& \times\left\langle\Phi(K \gamma)|H| \Phi\left(K^{\prime} \gamma^{\prime}\right)\right\rangle^{2}
\end{aligned}
$$

The summation in Eq. (2) is performed over all states $\gamma$ and $\gamma^{\prime}$ of the configurations $K$ and $K^{\prime}$, respectively. The $T\left(K, K^{\prime}\right) / g(K)$ is the average weight of the admixed configuration $K^{\prime}$ in the expansion of the wave functions for $K$. A singleconfiguration pseudo-relativistic method (Cowan 1981) is then applied to obtain radial orbitals for the corresponding configurations. The $\left\langle\Phi(K \gamma)|H| \Phi\left(K^{\prime} \gamma^{\prime}\right)\right\rangle$ is the interconfiguration matrix element of the pseudo-relativistic Hartree-Fock Hamiltonian $H$ (Cowan 1981). Previously, the same approach was succesfully applied to study energy levels (Radžiūtè et al. 2015), electric dipole (Kynienè et al. 2008) and magnetic (Jonauskas et al. 2010, 2012) transitions, and Auger cascades (Jonauskas et al. 2008, 2011; Palaudoux et al. 2010).

Single and double excitations from the considered configurations are studied to build the list of admixed configurations. The single excitations from the $2 \mathrm{~s}, 2 \mathrm{p}, 3 \mathrm{~s}, 3 \mathrm{p}$, and $3 d$ subshells up to subshells with $n=7$ and $l=5(l<n)$ are investigated in this work. However, promotion of electrons up to the subshells with $n=5$ and $l=4$ are considered for double excitations. The CIS values are calculated for all the produced configurations.

Direct and indirect processes have to be considered for the analysis of electron-impact single ionization from the level $i$ of $\mathrm{Fe}^{8+}$ to the level $f$ of $\mathrm{Fe}^{9+}$ :

$\sigma_{i f}(\varepsilon)=\sigma_{i f}^{\mathrm{DI}}(\varepsilon)+\sum_{k} \sigma_{i k}^{\mathrm{exc}}(\varepsilon) B_{k f}$

Here, $\varepsilon$ is the incident electron energy, $\sigma_{i f}^{\mathrm{DI}}$ represents the DI cross section, and $\sigma_{i k}^{\mathrm{exc}}$ corresponds to the electron-impact excitation cross section to the level $k$ of the $\mathrm{Fe}^{8+}$ ion. The summation over all final levels $f$ of $\mathrm{Fe}^{9+}$ in Eq. (3) produces the total single ionization cross sections for the level $i$. Electronimpact excitation and DI cross sections are studied using the DW approximation.

The radiative damping of the excited autoionizing levels of $\mathrm{Fe}^{8+}$ is included in the study by calculating the autoionization branching ratio which is determined by the expression

$B_{k j}=\frac{A_{k j}^{\mathrm{a}}}{\sum_{m} A_{k m}^{\mathrm{a}}+\sum_{n} A_{k n}^{\mathrm{r}}}$,

where $A^{\mathrm{a}}$ and $A^{\mathrm{r}}$ are the Auger and radiative transition probabilities, respectively.

For the ground configuration, DI includes ionization of the 3 s and 3 p subshells:

$3 s^{2} 3 p^{6}+e \rightarrow\left\{\begin{array}{l}3 s^{2} 3 p^{5}+2 e \\ 3 s^{1} 3 p^{6}+2 e\end{array}\right.$ 
The EA channels are investigated for the following excitations from the ground configuration,

$$
2 s^{2} 2 p^{6} 3 s^{2} 3 p^{6}+e \rightarrow\left\{\begin{array}{c}
2 s^{2} 2 p^{6} 3 s^{1} 3 p^{6} 3 d+e, \\
2 s^{2} 2 p^{6} 3 s^{1} 3 p^{6} n l+e \\
2 s^{2} 2 p^{5} 3 s^{2} 3 p^{6} 3 d+e \\
2 s^{2} 2 p^{5} 3 s^{2} 3 p^{6} n l+e \\
2 s^{1} 2 p^{6} 3 s^{2} 3 p^{6} 3 d+e \\
2 s^{1} 2 p^{6} 3 s^{2} 3 p^{6} n l+e
\end{array}\right.
$$

where $n \leqslant 20$ and $l \leqslant 6$. Therefore, 456 excited configurations are produced for which radiative and Auger decay processes are investigated. It should be noted that the excitations from the outermost subshell are not included in the study as the corresponding excited states are not autoionizing.

For the $3 s^{2} 3 p^{5} 3 d$ configuration, DI is analysed for the $3 s$, $3 \mathrm{p}$, and $3 \mathrm{~d}$ subshells:

$$
3 s^{2} 3 p^{6} 3 d+e \rightarrow\left\{\begin{array}{r}
3 s^{2} 3 p^{6}+2 e, \\
3 s^{2} 3 p^{5} 3 d+2 e, \\
3 s^{1} 3 p^{6} 3 d+2 e
\end{array}\right.
$$

The following excitations are studied to obtain the EA crosssections:

$$
2 s^{2} 2 p^{6} 3 s^{2} 3 p^{5} 3 d+e \rightarrow\left\{\begin{aligned}
2 s^{2} 2 p^{6} 3 s^{2} 3 p^{4} 3 d^{2} & +e, \\
2 s^{2} 2 p^{6} 3 s^{2} 3 p^{4} 3 d n l & +e, \\
2 s^{2} 2 p^{6} 3 s^{1} 3 p^{6} 3 d & +e, \\
2 s^{2} 2 p^{6} 3 s^{1} 3 p^{5} 3 d^{2} & +e, \\
2 s^{2} 2 p^{6} 3 s^{1} 3 p^{5} 3 d n l & +e, \\
2 s^{2} 2 p^{5} 3 s^{2} 3 p^{6} 3 d & +e, \\
2 s^{2} 2 p^{5} 3 s^{2} 3 p^{5} 3 d^{2} & +e, \\
2 s^{2} 2 p^{5} 3 s^{2} 3 p^{5} 3 d n l & +e, \\
2 s^{1} 2 p^{6} 3 s^{2} 3 p^{6} 3 d & +e, \\
2 s^{1} 2 p^{6} 3 s^{2} 3 p^{5} 3 d^{2} & +e, \\
2 s^{1} 2 p^{6} 3 s^{2} 3 p^{5} n l & +e .
\end{aligned}\right.
$$

This leads to 544 configurations.

The resonant excitation double autoionization (REDA) process is not considered in this study. The contribution from the REDA process is much smaller compared to DI and EA.

Electron-impact ionization cross sections $\sigma_{i j}(\varepsilon)$ are used to calculate the Maxwellian rate coefficients

$$
\begin{aligned}
\alpha_{i j}\left(T_{\mathrm{e}}\right)= & \left(\frac{1}{k_{\mathrm{B}} T_{\mathrm{e}}}\right)^{\frac{3}{2}}\left(\frac{8}{m_{\mathrm{e}} \pi}\right)^{\frac{1}{2}} \\
& \times \int_{0}^{\infty} \varepsilon \sigma_{i j}(\varepsilon) \exp \left(-\frac{\varepsilon}{k_{\mathrm{B}} T_{\mathrm{e}}}\right) \mathrm{d} \varepsilon
\end{aligned}
$$

for the Maxwellian-Boltzmann electron energy distribution at electron temperature $T_{\mathrm{e}}$. Here, $k_{\mathrm{B}}$ is the Boltzmann constant and $m_{\mathrm{e}}$ is the electron mass.

\section{Results}

The ground configuration of the $\mathrm{Fe}^{8+}$ ion is $[\mathrm{Ne}] 3 \mathrm{~s}^{2} 3 \mathrm{p}^{6}$. The energy levels for the ground configurations of the $\mathrm{Fe}^{8+}, \mathrm{Fe}^{9+}$, and $\mathrm{Fe}^{10+}$ ions are presented in Fig. 1. In addition, the main excited configurations of $\mathrm{Fe}^{8+}$ and $\mathrm{Fe}^{9+}$ are shown. These configurations play the main role in the formation of $\mathrm{Fe}^{9+}$ by electron impact. The single ionization threshold is $232.3 \mathrm{eV}$ while the energy provided by the National Institute of Standarts and Technology (NIST) equals $233.6 \mathrm{eV}$ (Kramida et al. 2018). The first excited configuration is formed by the $3 \mathrm{p} \rightarrow 3 \mathrm{~d}$ promotion from the ground configuration. The CI energy levels of the

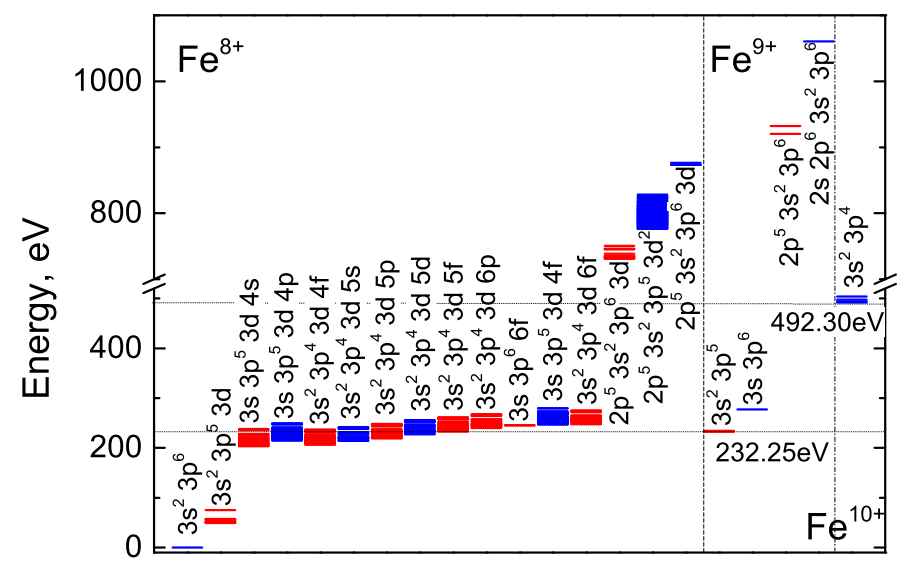

Fig. 1. Energy levels of the $\mathrm{Fe}^{8+}, \mathrm{Fe}^{9+}$, and $\mathrm{Fe}^{10+}$ ions. Blue (dark) represents even configurations; red (light) represents odd configurations.

first excited configuration varies from 50.730 to $73.893 \mathrm{eV}$ with respect to the ground level (Table 1). The CI leads to a diminishing of energy splitting from $25.566 \mathrm{eV}$ to $23.163 \mathrm{eV}$ for the energy levels of the $3 \mathrm{p}^{5} 3 \mathrm{~d}$ configuration.

The lifetimes for the energy levels of the first excited configuration are shown in Table 1 . The presented results correspond to the single and configuration interaction approximations. The CI basis is built by considering configurations that have the largest CIS values calculated from Eq. (2). The convergence for the lifetimes is determined by increasing the CI basis. Also, the $3 \mathrm{p}^{4} 3 \mathrm{~d}^{2}$, $3 \mathrm{p}^{4} 3 \mathrm{~d} 4 \mathrm{~d}$, and $3 \mathrm{~s} 3 \mathrm{p}^{6} 3 \mathrm{~d}$ configurations are studied since these configurations show the largest population transfer to $3 p^{5} 3 \mathrm{~d}$. In addition, configurations produced by radiative cascade from the admixed configurations are included in the CI basis. The total number of 20606 odd parity configuration state functions that originate from 62 configurations is included in the CI basis. For the even parity configurations, the CI basis consists of 17872 configuration state functions that are produced by 63 configurations.

Surprisingly, the lifetime of the ${ }^{3} \mathrm{~F}_{4}$ level demonstrates the largest change amongst those of the $3 \mathrm{p}^{5} 3 \mathrm{~d}$ configuration when correlation effects are included in the study. The lifetime of the level is diminished by factor of 2.6 from $1302.5 \mathrm{~s}$ to $493.2 \mathrm{~s}$. The transition probability from the level presented in the Chianti database (Dere et al. 1997; Landi et al. 2013) amounts to $9.22 \times$ $10^{-4} \mathrm{~s}^{-1}$ that corresponds to a lifetime of $1085 \mathrm{~s}$. On the other hand, the increasing of the CI lifetimes compared to the single configuration approximation is observed for many levels of the configuration. The largest effect is obtained for the ${ }^{1} \mathrm{P}_{1}$ level (index 12), which has the shortest lifetime. The admixed configurations change the lifetime by $36 \%$ for the level. Unfortunately, variation of the levels population over time due to radiative cascade does not show a large effect for the ${ }^{3} \mathrm{~F}_{4}$ level even for the extended CI basis when the time range of 15-50 s corresponding to the start and the end of data collection is considered. The population of the level varies in the range from $59 \%$ to $56 \%$. On the other hand, population modelling using data from single configuration calculations determines the fractional population of $66 \%$ and $64 \%$ for 15 and $50 \mathrm{~s}$, respectively. It should be noted that a lifetime of $493.2 \mathrm{~s}$ of the ${ }^{3} \mathrm{~F}_{4}$ level is larger compared to the collection time $(15-50 \mathrm{~s})$.

The ${ }^{3} \mathrm{~F}_{4}$ level can only decay to the ${ }^{3} \mathrm{P}_{2}$ level through E2 transitions. Our study, which includes 1844 configuration state functions (CSF) with $J=4$ and $2052 \mathrm{CSF}$ with $J=2$, leads to a 
Table 1. Energy levels (in eV) and lifetimes (in s) for the ground and $3 \mathrm{~s}^{2} 3 \mathrm{p}^{5} 3 \mathrm{~d}$ configurations of $\mathrm{Fe}^{8+}$.

\begin{tabular}{|c|c|c|c|c|c|c|c|}
\hline \multirow[b]{2}{*}{ Index } & \multirow[b]{2}{*}{ Term } & \multirow[b]{2}{*}{$J$} & \multicolumn{3}{|c|}{ Energy levels } & \multicolumn{2}{|c|}{ Lifetimes } \\
\hline & & & NIST & Single & CI & Single & $\mathrm{CI}$ \\
\hline 0 & $1 \mathrm{~S}$ & 0 & - & $-3.4027+4$ & $-3.4033+4$ & $\infty$ & $\infty$ \\
\hline 1 & $3 \mathrm{P}$ & 0 & $5.031+1$ & $4.962+1$ & $5.073+1$ & $\infty$ & $\infty$ \\
\hline 2 & $3 \mathrm{P}$ & 1 & $5.062+1$ & $4.994+1$ & $5.105+1$ & $6.34-8$ & $7.17-8$ \\
\hline 3 & $3 \mathrm{P}$ & 2 & $5.129+1$ & $5.062+1$ & $5.171+1$ & $1.23-2$ & $1.09-2$ \\
\hline 4 & $3 \mathrm{~F}$ & 4 & $5.279+1$ & $5.208+1$ & $5.347+1$ & $1.30+3$ & $4.93+2$ \\
\hline 5 & $3 \mathrm{~F}$ & 3 & $5.323+1$ & $5.254+1$ & $5.391+1$ & $7.42-1$ & $7.95-1$ \\
\hline 6 & $3 \mathrm{~F}$ & 2 & $5.379+1$ & $5.312+1$ & $5.447+1$ & $2.04-1$ & $1.98-1$ \\
\hline 7 & $3 \mathrm{D}$ & 3 & $5.649+1$ & $5.620+1$ & $5.729+1$ & $3.02-2$ & $3.26-2$ \\
\hline 8 & $3 \mathrm{D}$ & 1 & $5.711+1$ & $5.649+1$ & $5.749+1$ & $1.08-2$ & $1.05-2$ \\
\hline 9 & $3 \mathrm{D}$ & 2 & $5.736+1$ & $5.684+1$ & $5.790+1$ & $3.63-9$ & $3.69-9$ \\
\hline 10 & $1 \mathrm{D}$ & 2 & $5.663+1$ & $5.716+1$ & $5.816+1$ & $8.62-3$ & $9.76-3$ \\
\hline 11 & $1 \mathrm{~F}$ & 3 & $5.776+1$ & $5.746+1$ & $5.855+1$ & $6.77-3$ & $7.41-3$ \\
\hline 12 & $1 \mathrm{P}$ & 1 & $7.247+1$ & $7.519+1$ & $7.389+1$ & $3.08-12$ & $4.17-12$ \\
\hline
\end{tabular}

Notes. Absolute energies for the ground level are given for the single and CI calculations. NIST values are taken from Kramida et al. (2018). Note that $a \pm b \equiv a \times 10^{ \pm b}$.

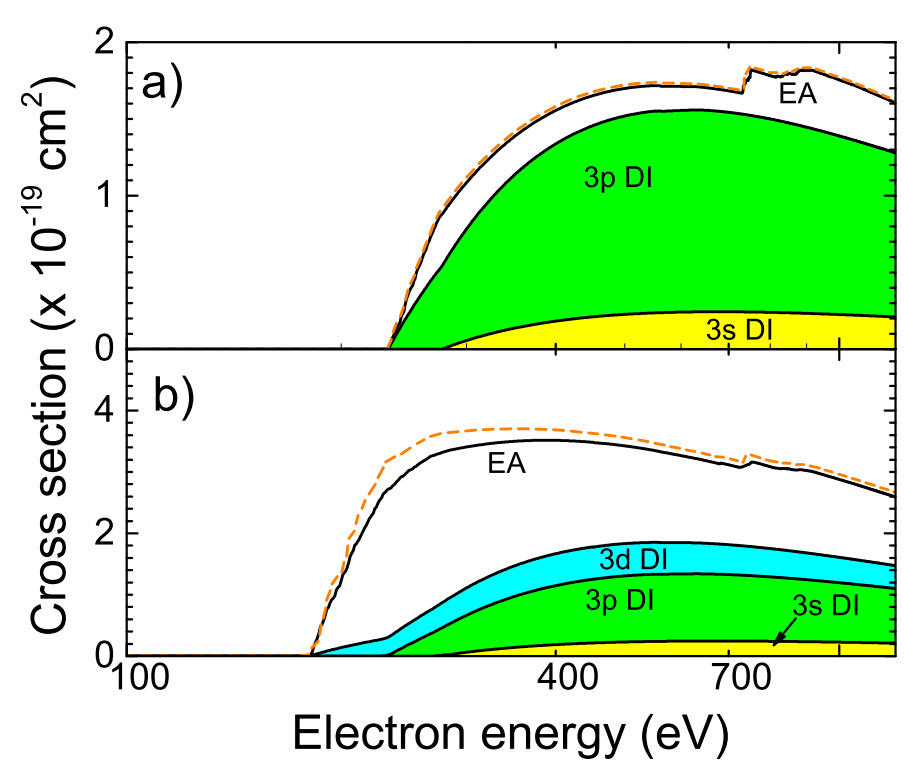

Fig. 2. Electron-impact ionization cross sections for a) ground $3 \mathrm{~s}^{2} 3 \mathrm{p}^{6}{ }^{1} \mathrm{~S}_{0}$ and $\mathrm{b}$ ) metastable $3 \mathrm{~s}^{2} 3 \mathrm{p}^{5} 3 \mathrm{~d}^{3} \mathrm{~F}_{4}$ levels of $\mathrm{Fe}^{8+}$. The SCADW data are presented by a dashed line for the total cross sections. The shaded areas show contributions from subshells for the DI process: $3 \mathrm{~s}$ (yellow), 3p (green), 3d (blue).

lifetime of 579.4 s. Since our CIS approach does not investigate Brillouin-type configurations, the basis of admixed configurations that in addition includes configurations produced by $n l \rightarrow$ $n^{\prime} l\left(n=2,3, l=0,1,2, n^{\prime}=3-10\right)$ excitations from the $3 \mathrm{p}^{5} 3 \mathrm{~d}$ configuration leads to $2447 \mathrm{CSF}$ with $J=4$ and $3239 \mathrm{CSF}$ with $J=2$. The lifetime for the ${ }^{3} \mathrm{~F}_{4}$ level decreases to $522.1 \mathrm{~s}$. Furthermore, an increase of the basis of the admixed configurations (without Brillouin-type configurations) up to $5393 \mathrm{CSF}$ with $J=4$ and $5935 \mathrm{CSF}$ with $J=2$ leads to 574.9 s for the level. Thus, the decrease of the lifetime from $579.4 \mathrm{~s}$ to $574.9 \mathrm{~s}$ is observed while CI bases changed from $3896(1844+2052)$ to $11328(5393+5935)$ CSF. As mentioned above, the extended basis of 20606 CSF that also includes Brillouin-type configurations gives $493.2 \mathrm{~s}$ for the lifetime. This suggests that a larger CI basis would not have a significant effect on the lifetime of the level.
The LLDW cross sections produced by electron impact ionization from the ground level and ${ }^{3} \mathrm{~F}_{4}$ level of the $3 \mathrm{~s}^{2} 3 \mathrm{p}^{5} 3 \mathrm{~d}$ configuration are presented in Fig. 2. The current study does not include the ${ }^{3} \mathrm{P}_{0}$ level since the population modelling in the time range $15-50 \mathrm{~s}$ provides a value of $\sim 1.8 \%$, which is lower than the fractional level population for the ground and ${ }^{3} \mathrm{~F}_{4}$ levels. A similar result for the first excited level of the $\mathrm{Fe}^{8+}$ ion was obtained by Hahn et al. (2016). For comparison, the sub-configuration average DW (SCADW) data are also shown. Good agreement amongst the LLDW and SCADW values can be seen. The obtained results demonstrate that cross sections for the ground level are approximately a factor of two lower compared to the ones from the ${ }^{4} \mathrm{~F}_{3}$ level. In addition, we have estimated cross sections for the direct double ionization (DDI) process in $\mathrm{Fe}^{8+}$ using a few-step approach (Jonauskas et al. 2014; Koncevičiūtè et al. 2018) The study of the electron-impact single ionization for the $\mathrm{Se}^{2+}$ (Koncevičiūtè et al. 2018) and $\mathrm{Se}^{3+}$ (Koncevičiūtè et al. 2019) ions determined that this process has a diminishing effect on the theoretical cross sections. However, the obtained cross sections for the DDI process in $\mathrm{Fe}^{8+}$ are an order of magnitude lower compared to the DI values. Thus, DDI does not provide any essential change in the theoretical single ionization cross sections.

Figure 2 shows that DI from the $3 p$ subshell dominates for both presented levels. The ionization from the $3 \mathrm{~s}$ subshell produces the lowest contribution. It should be mentioned that total DI cross sections for the ${ }^{4} \mathrm{~F}_{3}$ level are approximately $20 \%$ higher at the peak compared to the corresponding values of the ground level. The DI from the $2 \mathrm{~s}$ and $2 \mathrm{p}$ subshells mainly leads to double ionization of the $\mathrm{Fe}^{8+}$ ion through the ionization autoionization process and, therefore, is not considered in this study. The electron-impact ionization cross sections for the ground level are presented in Tables 2-5 and for the metastable level in Tables 6-10.

The contribution from the indirect process to the ionization cross sections for the metastable level leads to approximately two times higher total cross sections compared to the ground level. This can be explained by the fact that excitations from the $3 p$ subshell of the $3 s^{2} 3 p^{5} 3 d$ configuration provide strong EA channels. However, this subshell is the outermost one for the ground configuration and, thus, corresponding excitations do not have an effect on the EA cross sections. 
A. Kynienè et al.: Electron-impact ionization of $\mathrm{Fe}^{8+}$

Table 2. DI $3 s$ cross sections ( $\sigma$ in $\mathrm{Mb}$ ) of the ground level of $\mathrm{Fe}^{8+}$.

\begin{tabular}{cccccccc}
\hline \hline$\varepsilon$ & $\sigma$ & $\varepsilon$ & $\sigma$ & $\varepsilon$ & $\sigma$ & $\varepsilon$ & $\sigma$ \\
\hline $2.78+2$ & $2.69-1$ & $4.08+2$ & $1.88+1$ & $1.00+3$ & $2.26+1$ & $6.60+3$ & $6.62+0$ \\
$2.80+2$ & $8.29-1$ & $4.88+2$ & $2.24+1$ & $1.12+3$ & $2.16+1$ & $8.20+3$ & $5.56+0$ \\
$2.96+2$ & $4.80+0$ & $5.31+2$ & $2.33+1$ & $1.52+3$ & $1.86+1$ & $9.80+3$ & $4.80+0$ \\
$3.12+2$ & $8.03+0$ & $6.16+2$ & $2.42+1$ & $2.68+3$ & $1.31+1$ & $2.40+4$ & $2.21+0$ \\
$3.44+2$ & $1.29+1$ & $7.44+2$ & $2.42+1$ & $3.84+3$ & $1.01+1$ & $3.00+4$ & $1.82+0$ \\
$3.60+2$ & $1.48+1$ & $8.72+2$ & $2.35+1$ & $5.00+3$ & $8.25+0$ & $4.00+4$ & $1.43+0$ \\
\hline
\end{tabular}

Notes. Electron energies $\varepsilon$ are in $\mathrm{eV}$, and $a \pm b \equiv a \times 10^{ \pm b}$.

Table 3. DI $3 p$ cross sections ( $\sigma$ in $\mathrm{Mb}$ ) of the ground level of $\mathrm{Fe}^{8+}$.

\begin{tabular}{cccccccc}
\hline \hline$\varepsilon$ & $\sigma$ & $\varepsilon$ & $\sigma$ & $\varepsilon$ & $\sigma$ & $\varepsilon$ & $\sigma$ \\
\hline $2.33+2$ & $7.88-1$ & $3.60+2$ & $1.04+2$ & $1.00+3$ & $1.16+2$ & $8.20+3$ & $2.72+1$ \\
$2.40+2$ & $1.09+1$ & $4.05+2$ & $1.17+2$ & $1.12+3$ & $1.11+2$ & $9.80+3$ & $2.34+1$ \\
$2.80+2$ & $5.68+1$ & $4.88+2$ & $1.28+2$ & $1.52+3$ & $9.39+1$ & $2.00+4$ & $1.26+1$ \\
$2.96+2$ & $6.98+1$ & $5.40+2$ & $1.31+2$ & $2.68+3$ & $6.48+1$ & $2.40+4$ & $1.07+1$ \\
$3.12+2$ & $8.06+1$ & $6.16+2$ & $1.31+2$ & $3.84+3$ & $4.97+1$ & $2.80+4$ & $9.36+0$ \\
$3.28+2$ & $8.97+1$ & $7.44+2$ & $1.28+2$ & $5.00+3$ & $4.05+1$ & $3.20+4$ & $8.32+0$ \\
$3.44+2$ & $9.73+1$ & $8.72+2$ & $1.23+2$ & $6.60+3$ & $3.24+1$ & $4.00+4$ & $6.90+0$ \\
\hline
\end{tabular}

Notes. Electron energies $\varepsilon$ are in $\mathrm{eV}$, and $a \pm b \equiv a \times 10^{ \pm b}$.

Table 4. EA cross sections ( $\sigma$ in $\mathrm{Mb}$ ) of the ground level of $\mathrm{Fe}^{8+}$.

\begin{tabular}{cccccccc}
\hline \hline$\varepsilon$ & $\sigma$ & $\varepsilon$ & $\sigma$ & $\varepsilon$ & $\sigma$ & $\varepsilon$ & $\sigma$ \\
\hline $2.33+2$ & $2.97-1$ & $4.71+2$ & $1.88+1$ & $8.25+2$ & $2.88+1$ & $1.09+3$ & $3.49+1$ \\
$2.39+2$ & $3.67+0$ & $5.60+2$ & $1.64+1$ & $8.33+2$ & $2.94+1$ & $1.32+3$ & $3.06+1$ \\
$2.45+2$ & $6.17+0$ & $6.49+2$ & $1.48+1$ & $8.41+2$ & $3.14+1$ & $1.56+3$ & $2.79+1$ \\
$2.51+2$ & $1.44+1$ & $7.38+2$ & $1.97+1$ & $8.49+2$ & $3.24+1$ & $1.79+3$ & $2.59+1$ \\
$2.57+2$ & $2.05+1$ & $7.39+2$ & $2.05+1$ & $8.57+2$ & $3.22+1$ & $2.23+3$ & $2.31+1$ \\
$2.63+2$ & $2.48+1$ & $7.51+2$ & $2.80+1$ & $8.65+2$ & $3.25+1$ & $4.57+3$ & $1.57+1$ \\
$2.69+2$ & $3.00+1$ & $7.63+2$ & $2.94+1$ & $8.68+2$ & $3.26+1$ & $5.00+3$ & $1.48+1$ \\
$2.75+2$ & $3.40+1$ & $7.75+2$ & $2.90+1$ & $9.12+2$ & $3.76+1$ & $1.36+4$ & $8.02+0$ \\
$2.81+2$ & $3.30+1$ & $7.87+2$ & $2.86+1$ & $9.56+2$ & $3.73+1$ & $2.22+4$ & $5.87+0$ \\
$2.87+2$ & $3.20+1$ & $7.99+2$ & $2.82+1$ & $1.00+3$ & $3.65+1$ & $3.08+4$ & $4.89+0$ \\
$2.93+2$ & $3.11+1$ & $8.11+2$ & $2.80+1$ & $1.04+3$ & $3.59+1$ & $3.94+4$ & $4.17+0$ \\
$3.82+2$ & $2.28+1$ & $8.23+2$ & $2.89+1$ & $1.09+3$ & $3.49+1$ & $4.00+4$ & $4.13+0$ \\
\hline
\end{tabular}

Notes. Electron energies $\varepsilon$ are in $\mathrm{eV}$, and $a \pm b \equiv a \times 10^{ \pm b}$.

Table 5. Total cross sections ( $\sigma$ in $\mathrm{Mb}$ ) of the ground level of $\mathrm{Fe}^{8+}$.

\begin{tabular}{cccccccc}
\hline \hline$\varepsilon$ & $\sigma$ & $\varepsilon$ & $\sigma$ & $\varepsilon$ & $\sigma$ & $\varepsilon$ & $\sigma$ \\
\hline $2.33+2$ & $1.08+0$ & $3.86+2$ & $1.52+2$ & $7.77+2$ & $1.80+2$ & $3.64+3$ & $8.00+1$ \\
$2.37+2$ & $8.88+0$ & $4.12+2$ & $1.58+2$ & $8.11+2$ & $1.77+2$ & $4.46+3$ & $6.91+1$ \\
$2.46+2$ & $2.89+1$ & $4.38+2$ & $1.63+2$ & $8.45+2$ & $1.79+2$ & $4.87+3$ & $6.48+1$ \\
$2.55+2$ & $4.98+1$ & $4.85+2$ & $1.69+2$ & $8.79+2$ & $1.81+2$ & $7.80+3$ & $4.54+1$ \\
$2.64+2$ & $6.75+1$ & $5.29+2$ & $1.71+2$ & $9.13+2$ & $1.82+2$ & $1.12+4$ & $3.43+1$ \\
$2.73+2$ & $8.33+1$ & $5.73+2$ & $1.71+2$ & $1.11+3$ & $1.68+2$ & $1.68+4$ & $2.48+1$ \\
$2.82+2$ & $9.28+1$ & $6.17+2$ & $1.71+2$ & $1.69+3$ & $1.32+2$ & $2.24+4$ & $1.96+1$ \\
$3.08+2$ & $1.15+2$ & $6.61+2$ & $1.70+2$ & $1.88+3$ & $1.24+2$ & $2.80+4$ & $1.64+1$ \\
$3.34+2$ & $1.31+2$ & $7.09+2$ & $1.68+2$ & $2.77+3$ & $9.65+1$ & $3.36+4$ & $1.42+1$ \\
$3.60+2$ & $1.43+2$ & $7.43+2$ & $1.75+2$ & $3.23+3$ & $8.70+1$ & $4.00+4$ & $1.24+1$ \\
\hline
\end{tabular}

Notes. Electron energies $\varepsilon$ are in $\mathrm{eV}$, and $a \pm b \equiv a \times 10^{ \pm b}$. 
Table 6. DI $3 s$ cross sections ( $\sigma$ in $\mathrm{Mb}$ ) of the ${ }^{3} \mathrm{~F}_{4}$ level of $\mathrm{Fe}^{8+}$.

\begin{tabular}{cccccccc}
\hline \hline$\varepsilon$ & $\sigma$ & $\varepsilon$ & $\sigma$ & $\varepsilon$ & $\sigma$ & $\varepsilon$ & $\sigma$ \\
\hline $2.49+2$ & $9.21-2$ & $4.88+2$ & $2.27+1$ & $1.52+3$ & $1.87+1$ & $1.50+4$ & $3.35+0$ \\
$2.80+2$ & $2.02+0$ & $5.40+2$ & $2.38+1$ & $2.68+3$ & $1.31+1$ & $2.40+4$ & $2.21+0$ \\
$2.96+2$ & $5.44+0$ & $6.16+2$ & $2.45+1$ & $3.84+3$ & $1.01+1$ & $2.40+4$ & $2.21+0$ \\
$3.28+2$ & $1.13+1$ & $7.44+2$ & $2.45+1$ & $5.00+3$ & $8.26+0$ & $2.70+4$ & $2.00+0$ \\
$3.44+2$ & $1.35+1$ & $8.72+2$ & $2.37+1$ & $6.60+3$ & $6.64+0$ & $3.00+4$ & $1.82+0$ \\
$3.60+2$ & $1.53+1$ & $1.00+3$ & $2.27+1$ & $8.20+3$ & $5.57+0$ & $3.60+4$ & $1.55+0$ \\
$3.98+2$ & $1.86+1$ & $1.12+3$ & $2.18+1$ & $9.80+3$ & $4.81+0$ & $4.00+4$ & $1.43+0$ \\
\hline
\end{tabular}

Notes. Electron energies $\varepsilon$ are in $\mathrm{eV}$, and $a \pm b \equiv a \times 10^{ \pm b}$.

Table 7. DI $3 \mathrm{p}$ cross sections ( $\sigma$ in $\mathrm{Mb}$ ) of the ${ }^{3} \mathrm{~F}_{4}$ level of $\mathrm{Fe}^{8+}$.

\begin{tabular}{cccccccc}
\hline \hline$\varepsilon$ & $\sigma$ & $\varepsilon$ & $\sigma$ & $\varepsilon$ & $\sigma$ & $\varepsilon$ & $\sigma$ \\
\hline $2.40+2$ & $9.36+0$ & $4.05+2$ & $9.71+1$ & $1.16+3$ & $9.06+1$ & $8.20+3$ & $2.26+1$ \\
$2.61+2$ & $3.10+1$ & $5.10+2$ & $1.08+2$ & $1.62+3$ & $7.53+1$ & $9.80+3$ & $1.95+1$ \\
$2.82+2$ & $4.84+1$ & $5.54+2$ & $1.09+2$ & $2.46+3$ & $5.73+1$ & $2.50+4$ & $8.62+0$ \\
$3.03+2$ & $6.20+1$ & $5.98+2$ & $1.09+2$ & $3.30+3$ & $4.63+1$ & $2.80+4$ & $7.79+0$ \\
$3.24+2$ & $7.28+1$ & $6.72+2$ & $1.09+2$ & $4.14+3$ & $3.90+1$ & $3.10+4$ & $7.12+0$ \\
$3.45+2$ & $8.12+1$ & $8.34+2$ & $1.04+2$ & $5.00+3$ & $3.37+1$ & $3.40+4$ & $6.57+0$ \\
$3.48+2$ & $8.23+1$ & $9.96+2$ & $9.71+1$ & $6.60+3$ & $2.70+1$ & $4.00+4$ & $5.73+0$ \\
\hline
\end{tabular}

Notes. Electron energies $\varepsilon$ are in $\mathrm{eV}$, and $a \pm b \equiv a \times 10^{ \pm b}$.

Table 8. DI $3 \mathrm{~d}$ cross sections $\left(\sigma\right.$ in $\mathrm{Mb}$ ) of the ${ }^{3} F_{4}$ level of $\mathrm{Fe}^{8+}$.

\begin{tabular}{cccccccc}
\hline \hline$\varepsilon$ & $\sigma$ & $\varepsilon$ & $\sigma$ & $\varepsilon$ & $\sigma$ & $\varepsilon$ & $\sigma$ \\
\hline $1.81+2$ & $6.62-1$ & $3.52+2$ & $4.98+1$ & $8.34+2$ & $4.53+1$ & $6.90+3$ & $9.87+0$ \\
$1.97+2$ & $1.21+1$ & $3.97+2$ & $5.19+1$ & $1.01+3$ & $4.12+1$ & $6.90+3$ & $9.87+0$ \\
$2.13+2$ & $2.09+1$ & $4.42+2$ & $5.27+1$ & $1.18+3$ & $3.77+1$ & $8.10+3$ & $8.61+0$ \\
$2.29+2$ & $2.77+1$ & $4.87+2$ & $5.27+1$ & $2.71+3$ & $2.11+1$ & $9.30+3$ & $7.65+0$ \\
$2.45+2$ & $3.31+1$ & $4.90+2$ & $5.27+1$ & $3.33+3$ & $1.80+1$ & $1.50+4$ & $5.00+0$ \\
$2.62+2$ & $3.76+1$ & $5.91+2$ & $5.12+1$ & $3.95+3$ & $1.57+1$ & $2.70+4$ & $2.90+0$ \\
$3.07+2$ & $4.55+1$ & $6.62+2$ & $4.96+1$ & $4.57+3$ & $1.39+1$ & $4.00+4$ & $2.02+0$ \\
\hline
\end{tabular}

Notes. Electron energies $\varepsilon$ are in $\mathrm{eV}$, and $a \pm b \equiv a \times 10^{ \pm b}$.

Surprisingly, the excitation from the $2 \mathrm{p}$ to the $3 \mathrm{~d}$ subshell with subsequent autoionization produces the largest contribution to the EA process for the ground level (Fig. 3). The threshold for this excitation is $731 \mathrm{eV}$. The contribution from the $2 \mathrm{p}$ subshell excitations is clearly seen in the total cross sections starting from this energy (Fig. 2a). The same transition was identified as the strongest EA channel for the ground and excited $3 s^{2} 3 p^{5} 3 d$ configurations using the CADW method (Pindzola et al. 1987). It should be noted that the EA $3 \mathrm{~s} \rightarrow$ of channel is the strongest one at the lower energies of the incident electron (Fig. 3). Furthermore, the total EA cross sections are approximately $30 \%$ higher for the $3 \mathrm{~s}$ subshell compared to the excitations from the $2 \mathrm{p}$ subshell (Fig. 3). It can be seen that the excitations to many autoionizing configurations have to be considered to reach convergence for the $2 \mathrm{p}$ and $3 \mathrm{~s}$ subshells.

The situation drastically changes for the EA cross sections corresponding to electron-impact ionization from the ${ }^{3} \mathrm{~F}_{4}$ level of the $3 p^{5} 3 \mathrm{~d}$ configuration (Fig. 4). The contribution from the $2 p$ subshell is considerably lower compared to the excitations from the $3 p$ subshell. The five strongest EA channels correspond to the $3 p \rightarrow 5 f, 3 p \rightarrow 6 p, 3 p \rightarrow 6 f, 3 s \rightarrow 4 f$, and $2 p \rightarrow 3 d$ excitations. The next strongest excitation after the $2 \mathrm{p}$ subshell occurs to the $4 \mathrm{~d}$ subshell. However, its influence is an order of magnitude lower compared to $2 \mathrm{p} \rightarrow 3 \mathrm{~d}$.

The EA channels corresponding to excitations from the ground level to the subshells with orbital quantum number $l=3$ dominate at the lower energies of the incident electron (Fig. 5). The $3 \mathrm{~s}$ subshell participates in the formation of the EA cross sections in the energy region from the threshold up to $\sim 730 \mathrm{eV}$ where the EA $2 p$ channel opens. The excitations from $2 p$ to $l=2$ make the largest contribution at the higher energies. The $2 p \rightarrow 3 d$ excitation provides $\sim 50 \%$ of the total cross sections for the peak values in this energy region. We see that radiative damping has a crucial effect on the EA values corresponding to the excitations to $l=0$ at the lower energy side. However, the configurations produced by the excitations from the $2 \mathrm{~s}$ and $2 \mathrm{p}$ subshells mainly decay to the next ionization stage without a significant effect from the radiative damping.

The excitations to orbital quantum number $l=3$ produce the largest contribution to the EA cross sections for the metastable level in all energy ranges (Fig. 6). For the excitations up to subshells with $n=8$, it gives approximately half of the total cross sections (Fig. 4). For the higher shells, the excitations to the subshells with $l=3$ also dominate but with the lower weight. 
Table 9. EA cross sections $\left(\sigma\right.$ in $\mathrm{Mb}$ ) of the ${ }^{3} \mathrm{~F}_{4}$ level of $\mathrm{Fe}^{8+}$.

\begin{tabular}{cccccccc}
\hline \hline$\varepsilon$ & $\sigma$ & $\varepsilon$ & $\sigma$ & $\varepsilon$ & $\sigma$ & $\varepsilon$ & $\sigma$ \\
\hline $1.81+2$ & $1.28+0$ & $2.80+2$ & $2.43+2$ & $7.40+2$ & $1.32+2$ & $3.28+3$ & $5.55+1$ \\
$1.89+2$ & $5.11+1$ & $2.96+2$ & $2.32+2$ & $7.71+2$ & $1.37+2$ & $4.00+3$ & $4.81+1$ \\
$1.97+2$ & $9.59+1$ & $3.61+2$ & $1.99+2$ & $7.99+2$ & $1.33+2$ & $4.69+3$ & $4.28+1$ \\
$2.05+2$ & $1.37+2$ & $4.26+2$ & $1.77+2$ & $8.27+2$ & $1.32+2$ & $9.00+3$ & $2.61+1$ \\
$2.13+2$ & $1.75+2$ & $4.91+2$ & $1.61+2$ & $8.55+2$ & $1.33+2$ & $1.26+4$ & $2.01+1$ \\
$2.21+2$ & $2.05+2$ & $5.56+2$ & $1.49+2$ & $8.83+2$ & $1.34+2$ & $2.60+4$ & $1.19+1$ \\
$2.24+2$ & $2.17+2$ & $6.24+2$ & $1.38+2$ & $9.11+2$ & $1.34+2$ & $2.88+4$ & $1.11+1$ \\
$2.38+2$ & $2.42+2$ & $6.53+2$ & $1.34+2$ & $1.16+3$ & $1.15+2$ & $3.16+4$ & $1.05+1$ \\
$2.52+2$ & $2.48+2$ & $6.82+2$ & $1.32+2$ & $1.64+3$ & $9.00+1$ & $3.44+4$ & $9.97+0$ \\
$2.66+2$ & $2.50+2$ & $7.11+2$ & $1.29+2$ & $1.88+3$ & $8.19+1$ & $4.00+4$ & $9.09+0$ \\
\hline
\end{tabular}

Notes. Electron energies $\varepsilon$ are in $\mathrm{eV}$, and $a \pm b \equiv a \times 10^{ \pm b}$.

Table 10. Total cross sections $\left(\sigma\right.$ in $\mathrm{Mb}$ ) of the ${ }^{3} \mathrm{~F}_{4}$ level of $\mathrm{Fe}^{8+}$.

\begin{tabular}{cccccccc}
\hline \hline$\varepsilon$ & $\sigma$ & $\varepsilon$ & $\sigma$ & $\varepsilon$ & $\sigma$ & $\varepsilon$ & $\sigma$ \\
\hline $1.81+2$ & $1.94+0$ & $2.66+2$ & $3.24+2$ & $8.22+2$ & $3.06+2$ & $3.53+3$ & $1.25+2$ \\
$1.86+2$ & $3.71+1$ & $2.76+2$ & $3.31+2$ & $8.79+2$ & $3.04+2$ & $4.01+3$ & $1.13+2$ \\
$1.94+2$ & $9.14+1$ & $2.86+2$ & $3.36+2$ & $9.36+2$ & $2.98+2$ & $4.49+3$ & $1.04+2$ \\
$2.02+2$ & $1.36+2$ & $3.47+2$ & $3.50+2$ & $9.94+2$ & $2.89+2$ & $4.97+3$ & $9.61+1$ \\
$2.10+2$ & $1.83+2$ & $4.08+2$ & $3.51+2$ & $1.17+3$ & $2.63+2$ & $9.70+3$ & $5.66+1$ \\
$2.18+2$ & $2.18+2$ & $4.69+2$ & $3.46+2$ & $1.52+3$ & $2.23+2$ & $1.44+4$ & $4.09+1$ \\
$2.26+2$ & $2.52+2$ & $5.30+2$ & $3.38+2$ & $1.70+3$ & $2.08+2$ & $1.94+4$ & $3.19+1$ \\
$2.36+2$ & $2.77+2$ & $6.51+2$ & $3.18+2$ & $1.88+3$ & $1.95+2$ & $2.44+4$ & $2.65+1$ \\
$2.46+2$ & $2.96+2$ & $7.08+2$ & $3.10+2$ & $2.57+3$ & $1.57+2$ & $2.94+4$ & $2.30+1$ \\
$2.56+2$ & $3.11+2$ & $7.65+2$ & $3.15+2$ & $3.05+3$ & $1.39+2$ & $4.00+4$ & $1.82+1$ \\
\hline
\end{tabular}

Notes. Electron energies $\varepsilon$ are in $\mathrm{eV}$, and $a \pm b \equiv a \times 10^{ \pm b}$.

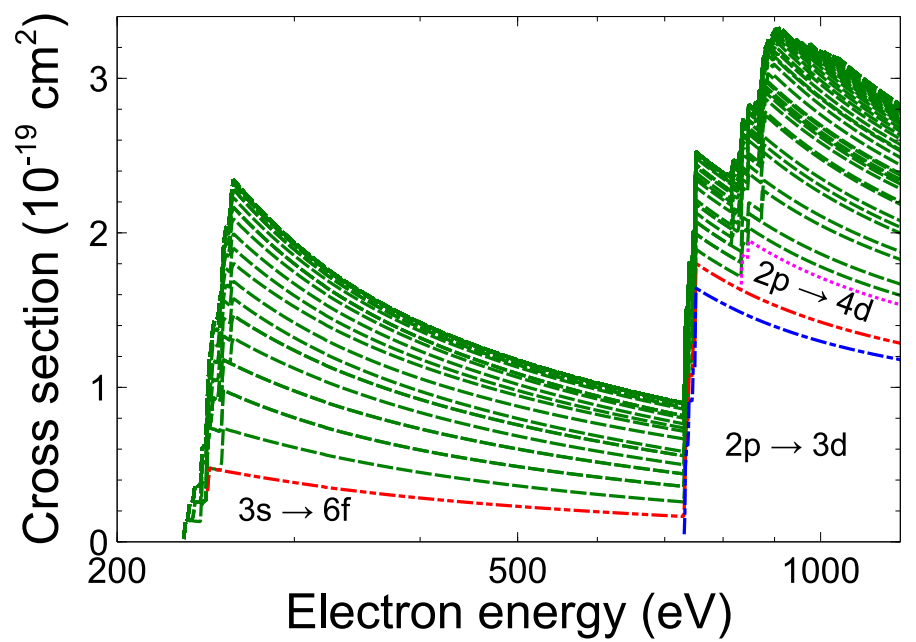

Fig. 3. Strongest EA channels for the ground level of $\mathrm{Fe}^{8+}$ for excitations to shells with $n \leqslant 8$ : dashed-dotted (blue) line: $2 \mathrm{p} \rightarrow 3 \mathrm{~d}$, dasheddotted-dotted (red): $3 s \rightarrow 6 f$, dotted (magenta): $2 \mathrm{p} \rightarrow 4 \mathrm{~d}$.

Similar results are also obtained for the SCADW values. However, the excitations to the subshells with $l=1$ provide the second largest contribution in contrast to the results obtained for the ground level. This can be explained by the fact that promotion of the electron from the $3 p$ subshell leads to autoionizing states for the metastable level. The radiative damping diminishes the EA cross sections for the strongest channels up to $20 \%$.

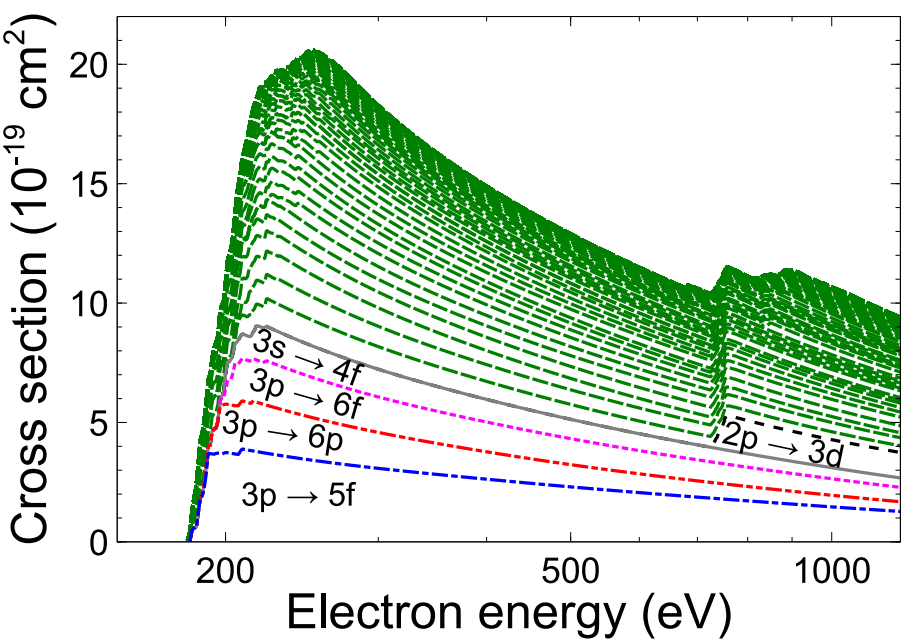

Fig. 4. Strongest EA channels for the metastable ${ }^{3} \mathrm{~F}_{4}$ level of $\mathrm{Fe}^{8+}$ for excitations to shells with $n \leqslant 8$ : dashed-dotted (blue) line: $3 \mathrm{p} \rightarrow 5 \mathrm{f}$, dashed-dotted-dotted (red): $3 p \rightarrow 6 p$, dotted (magenta): $3 p \rightarrow 6 f$, solid (grey): $3 \mathrm{~s} \rightarrow 4 \mathrm{f}$, long-dashed (black): $2 \mathrm{p} \rightarrow 3 \mathrm{~d}$.

It is important to ensure convergence of the EA cross sections due to excitations to the high- $n l$ subshells. It was demonstrated that excitations to the high- $n l$ subshells can have a crucial effect for the tungsten ions (Zhang \& Kwon 2014; Jonauskas et al. 2015a; Kynienè et al. 2015, 2016). Figure 7 demonstrates the changes of the ${ }^{3} \mathrm{~F}_{4}$ EA cross sections for the excitations from the $3 \mathrm{~s}$ and $3 \mathrm{p}$ subshells up to shells with $n=5,10$, and 20. The 


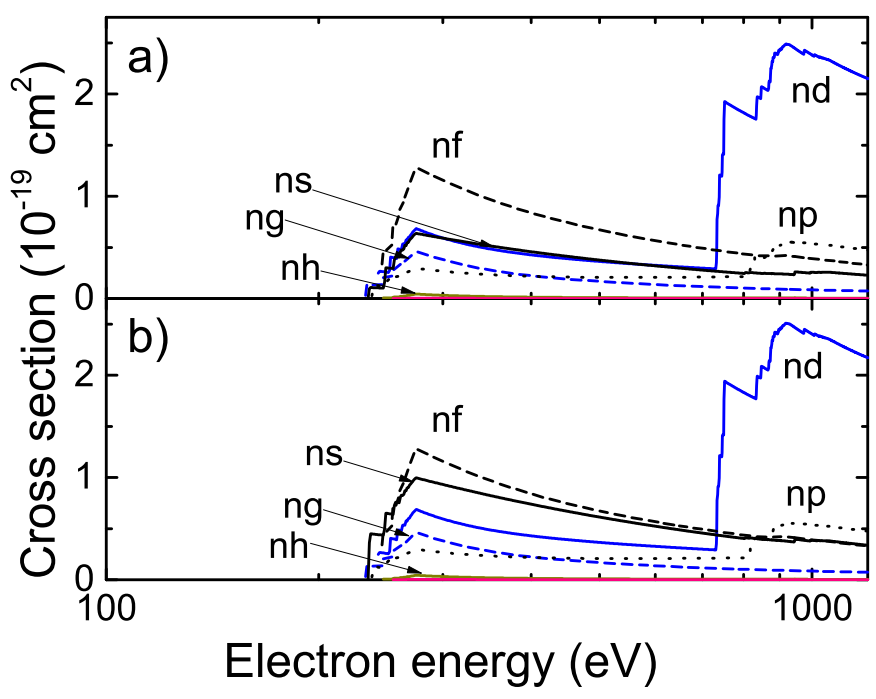

Fig. 5. Contribution of the EA channels corresponding to excitations from the ground level to shells with different orbital quantum numbers with (panel $a$ ) and without (panel $b$ ) radiative damping.

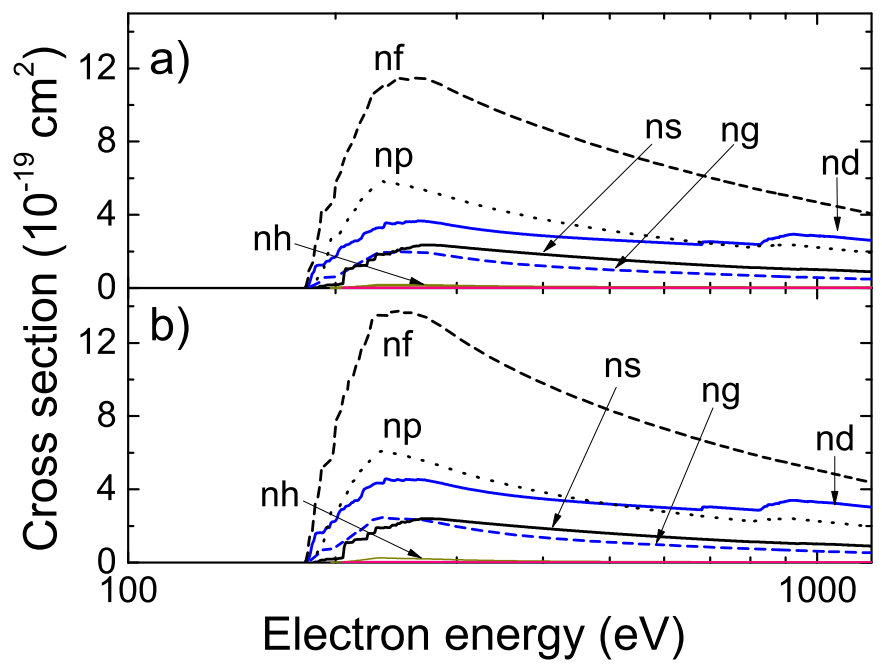

Fig. 6. Contribution of the EA channels corresponding to excitations from the metastable ${ }^{3} \mathrm{~F}_{4}$ level to shells with different orbital quantum numbers with (panel $a$ ) and without (panel $b$ ) radiative damping.

presented data show that the excitations to the shells with $n>10$ increase the EA cross sections by $\sim 7 \%$ for the $3 \mathrm{~s}$ subshell and $\sim 12 \%$ for the $3 \mathrm{p}$ subshell. The influence of the excitations to the higher shells $(20<n \leqslant 40)$ is studied using the SCADW approach. The determined contribution to the total cross sections is lower than $2 \%$ for the ground and ${ }^{3} \mathrm{~F}_{4}$ levels.

The excited configurations can also decay through radiative transitions. This leads to radiative damping of the EA cross sections. The radiative damping diminishes the total EA cross sections for the metastable level by $\sim 12 \%$ for the 3 s subshell and $\sim 15 \%$ for the $3 p$ subshell (Fig. 7). The influence of the radiative damping on the EA process from the $3 \mathrm{~s}$ subshell is lower than $11 \%$ for the ground level. It should be noted that the radiative damping is less important for the total ionization cross sections of the ground level compared to the metastable one, since the DI part dominates in the first case.

Our obtained values for ionization from the ground and metastable $3 \mathrm{~s}^{2} 3 \mathrm{p}^{5} 3 \mathrm{~d}^{3} \mathrm{~F}_{4}$ levels are compared with experimental data in Fig. 8. It can be seen that better agreement with mea-

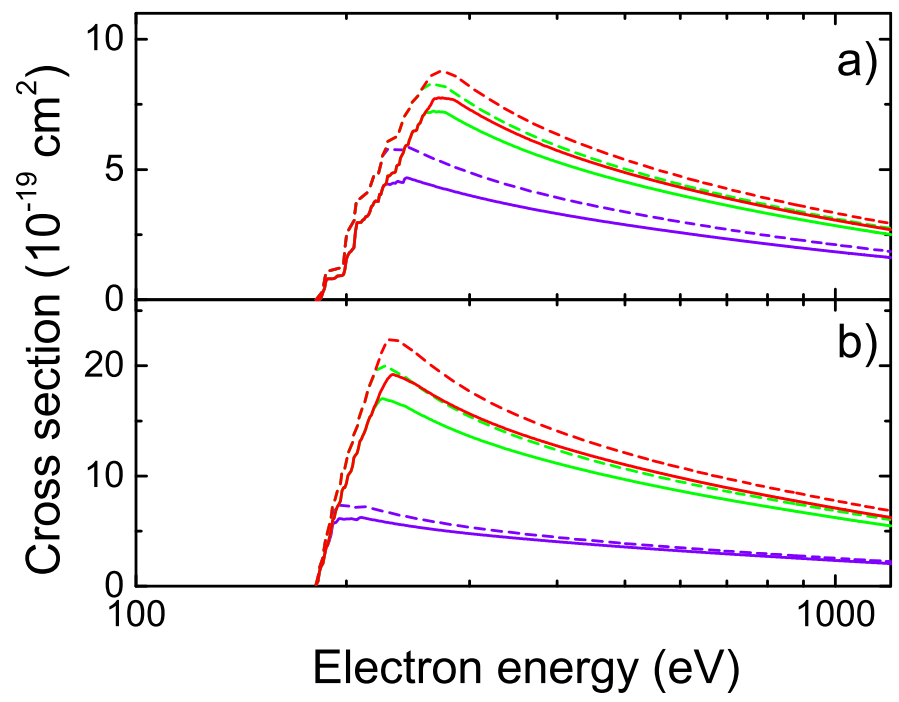

Fig. 7. Contribution of excitations to the shells with $n \leqslant 5$ (blue), $n \leqslant 10$ (green), and $n \leqslant 20$ (red) from the $3 \mathrm{~s}$ (panel $a$ ) and 3 p (panel $b$ ) subshells for the metastable ${ }^{3} \mathrm{~F}_{4}$ level. The cross sections calculated without radiative damping are represented by dashed lines.

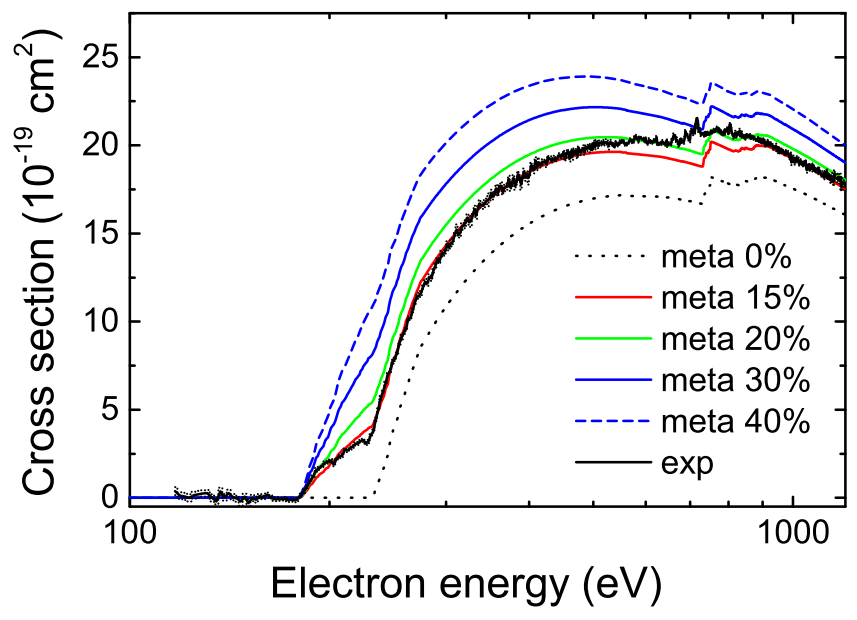

Fig. 8. Comparison of theoretical cross sections with experimental values. The metastable fraction is shown by the different lines.

surements for electron energies up to $500 \mathrm{eV}$ and above $900 \mathrm{eV}$ occurs when $15 \%$ of the ions in the beam reside in the metastable state. However, these values are below the experimental data for the peak energies but still within the error bars. The previous calculations using the CADW approach determined $\sim 30 \%$ for the metastable fraction. It should be noted that CADW calculations (Hahn et al. 2016) provide $\sim 40 \%$ higher cross sections compared to the LLDW values for the ground level. On the other hand, our cross sections are only $\sim 10 \%$ above the CADW ones for the ${ }^{3} \mathrm{~F}_{4}$ level. However, the previous calculations included only $3 \mathrm{~s} \rightarrow 41$ transitions for the $3 s^{2} 3 p^{5} 3 \mathrm{~d}$ configuration (Hahn et al. 2016). Our presented results demonstrate a very strong contribution from the excitations to shells with $n=5$ and $n=6$ (Fig. 5). Many of the configurations produced by these excitations are just above, or their energy levels straddle, the ionization threshold (Fig. 1). This leads to the high values for the EA process. Therefore, the full CADW calculations for the metastable level would produce a much higher contribution from the ground level in the fitting procedure. This would lead to much lower values for the metastable fraction. 
A. Kynienė et al.: Electron-impact ionization of $\mathrm{Fe}^{8+}$

Table 11. Maxwellian rate coefficients for the $3 \mathrm{~s}^{2} 3 \mathrm{p}^{6}{ }^{1} \mathrm{~S}_{0}$ and the $3 \mathrm{~s}^{2} 3 \mathrm{p}^{5} 3 \mathrm{~d}^{3} \mathrm{~F}_{4}$ levels in $\mathrm{cm}^{3} \mathrm{~s}^{-1}$.

\begin{tabular}{cccccccc}
\hline \hline & \multicolumn{3}{c}{$3 \mathrm{~s}^{2} 3 \mathrm{p}^{6}{ }^{1} \mathrm{~S}_{0}$} & & \multicolumn{3}{c}{$3 \mathrm{~s}^{2} 3 \mathrm{p}^{5} 3 \mathrm{~d}^{3} \mathrm{~F}_{4}$} \\
\cline { 2 - 4 } \cline { 6 - 8 }$T_{e}$ & DI & EA & Total & & DI & EA & Total \\
\hline $8+5$ & $6.200-17$ & $1.830-17$ & $8.030-17$ & & $9.831-17$ & $2.652-16$ & $3.635-16$ \\
$2+6$ & $6.202-16$ & $1.192-16$ & $7.396-16$ & & $7.994-16$ & $1.113-15$ & $1.913-15$ \\
$4+6$ & $1.358-15$ & $2.613-16$ & $1.622-15$ & & $1.648-15$ & $1.674-15$ & $3.325-15$ \\
$8+6$ & $1.948-15$ & $4.259-16$ & $2.377-15$ & & $2.291-15$ & $1.959-15$ & $4.253-15$ \\
$2+7$ & $2.188-15$ & $5.576-16$ & $2.750-15$ & & $2.514-15$ & $1.945-15$ & $4.468-15$ \\
$4+7$ & $2.028-15$ & $5.833-16$ & $2.632-15$ & & $2.306-15$ & $1.767-15$ & $4.100-15$ \\
$8+7$ & $1.733-15$ & $5.679-16$ & $2.331-15$ & & $1.955-15$ & $1.537-15$ & $3.527-15$ \\
$2+8$ & $1.171-15$ & $4.385-16$ & $1.625-15$ & & $1.312-15$ & $1.079-15$ & $2.408-15$ \\
$4+8$ & $6.718-16$ & $2.643-16$ & $9.406-16$ & & $7.507-16$ & $6.303-16$ & $1.386-15$ \\
$8+8$ & $3.142-16$ & $1.266-16$ & $4.416-16$ & & $3.506-16$ & $2.976-16$ & $6.492-16$ \\
$2+9$ & $9.519-17$ & $3.887-17$ & $1.341-16$ & & $1.062-16$ & $9.066-17$ & $1.969-16$ \\
$4+9$ & $3.582-17$ & $1.469-17$ & $5.048-17$ & & $3.994-17$ & $3.417-17$ & $7.409-17$ \\
\hline
\end{tabular}

Notes. Temperatures $\left(T_{e}\right)$ are given in K. Note that $a \pm b \equiv a \times 10^{ \pm b}$.

It should be noted that the obtained data contradict the results determined from the population modelling using the Boltzmann distribution. This suggests that other mechanisms of the level population have to be considered. Further studies could include a full collisional-radiative model for the interaction regime to define the initial level population for the subsequent radiative cascade. The time-dependent modelling of the radiative cascade would be performed from the last injection to the end of data collection. The similar approach used before demonstrated different intensities for spectral lines compared to the ones obtained only from the collisional-radiative model (Jonauskas et al. 2013, 2015b). What is more, the lower value of the lifetime for the ${ }^{3} \mathrm{~F}_{4}$ level would lead to the lower fractional population. The calculations show that the lifetime would have to be lower by approximately an order of magnitude than the currently used value so that it would correspond to the population of the level determined from cross section comparison. However, it is highly doubtful that the larger CI basis would lead to significant change in the lifetime.

It should be noted that calculations of the cross sections using the DW approximation usually provide a good agreement with measurements for the highly ionized ions (Loch et al. 2005; Kwon \& Savin 2012; Zhang \& Kwon 2014; Borovik et al. 2015). The $\mathrm{Fe}^{8+}$ ion should not be the exception. This means that additional measurements may help to resolve the disagreement between the different methods. On the other hand, more sophisticated calculations for the lifetimes of the energy levels of the $3 s^{2} 3 p^{5} 3 d$ configuration are also needed.

Finally, the Maxwellian rate coefficients for the ground and metastable levels are presented in Table 11. The data are shown for the DI, EA, and total ionization.

\section{Conclusions}

Electron-impact ionization cross sections have been studied for the ground and metastable $3 \mathrm{~s}^{2} 3 \mathrm{p}^{5} 3 \mathrm{~d}^{3} \mathrm{~F}_{4}$ levels of the $\mathrm{Fe}^{8+}$ ion. Convergence for the EA cross sections due to excitations to the high- $n l$ subshells has been investigated. The cross sections for the metastable level are approximately a factor of two higher compared to values for the ground level.

The study demonstrates that excitations to subshells with orbital quantum number $l=3$ dominate for the ground and metastable levels of the $\mathrm{Fe}^{8+}$ ion at the lower energy side. For the ground level at energies above $731 \mathrm{eV}$, the $2 p$ subshell opens, which leads to the strong increase of the EA cross sections due to excitations to the subshells with $l=2$.

The presented results are compared with the recent TSR measurements. Modelling of theoretical cross sections for the ground and metastable levels to produce the best fit to measurements shows that $15 \%$ of the ions in the ion beam reside in the metastable state. On the other hand, modelling of the levels population over time using the Boltzmann distribution for the initial population provides $59 \%-50 \%$ for the metastable level. This suggests that other mechanisms of the levels population have to be considered. On the other hand, the radiative decay processes have to be analysed for energy levels of the $3 \mathrm{~s}^{2} 3 \mathrm{p}^{5} 3 \mathrm{~d}$ configuration using the larger CI bases or other approaches.

Radiative decay from the levels of the $3 s^{2} 3 p^{5} 3 d$ configuration are investigated using the extended CI basis. The correlation effects diminish the lifetime of the $3 \mathrm{p}^{5} 3 \mathrm{~d}^{3} \mathrm{~F}_{4}$ level by approximately three times from $1302 \mathrm{~s}$ to $493 \mathrm{~s}$ compared to calculations from the single configuration approximation.

Acknowledgements. Part of the computations were performed on resources at the High Performance Computing Center "HPC Sauletekis" at Vilnius University, Faculty of Physics.

\section{References}

Bernhardt, D., Becker, A., Grieser, M., et al. 2014, Phys. Rev. A, 90, 012702 Beiersdorfer, P., Lepson, J. K., Desai, P., Díaz, F., \& Ishikawa, Y. 2014, ApJS, 210, 16

Borovik, Jr., A. B., Gharaibeh, M. F., Schippers, S., \& Müller, A. 2015, J. Phys. B: At. Mol. Opt. Phys., 48, 035203

Butler, K., \& Moores, D. L. 1985, J. Phys. B: At. Mol. Opt. Phys., 18, 1247

Cowan, R. D. 1981, The Theory of Atomic Structure and Spectra (Berkeley, CA: University of California Press)

Dere, K. P. 2007, A\&A, 466, 771

Dere, K. P., Landi, E., Mason, H. E., Monsignori Fossi, B. C., \& Young, P. R. 1997, A\&AS, 125, 149

Freund, R. S., Robert, C., Shul, R. J., et al. 1990, Phys. Rev. A, 41, 7

Gabriel, A. H., Fawcett, B. C., \& Jordan, C. 1966, Proc. Phys. Soc., 87, 825

Gregory, D. C., Meyer, F. W., Müller, A., \& Defrance, P. 1986, Phys. Rev. A, 34, 3657

Gregory, D. C., Wang, L. J., Meyer, F. W., \& Rinn, K. 1987, Phys. Rev. A, 35, 3256

Gu, M. F. 2008, Can. J. Phys., 86, 675

Hahn, M., Grieser, M., Krantz, C., et al. 2011, ApJ, 735, 105

Hahn, M., Becker, A., Grieser, M., et al. 2012, ApJ, 760, 80 
Hahn, M., Becker, A., Bernhardt, D., et al. 2013, ApJ, 767, 47

Hahn, M., Becker, A., Bernhardt, D., et al. 2015, ApJ, 813, 16

Hahn, M., Becker, A., Bernhardt, D., et al. 2016, J. Phys. B: At. Mol. Opt. Phys., 49, 084006

Hummer, D. G., Berrington, K. A., Eissner, W., et al. 1993, A\&A, 279, 298

Jonauskas, V., Karazija, R., \& Kučas, S. 2008, J. Phys. B: At. Mol. Opt. Phys., 41, 215005 (5pp)

Jonauskas, V., Kisielius, R., Kynienė, A., Kučas, S., \& Norrington, P. H. 2010, Phys. Rev. A, 81, 012506

Jonauskas, V., Kučas, S., \& Karazija, R. 2011, Phys. Rev. A, 84, 053415

Jonauskas, V., Gaigalas, G., \& Kučas, S. 2012, Atom. Data Nucl. Data, 98, 19

Jonauskas, V., Masys, Š., Kynienè, A., \& Gaigalas, G. 2013, J. Quant. Spectr. Rad. Transf., 127, 64

Jonauskas, V., Prancikevičius, A., Masys, Š., \& Kynienè, A. 2014, Phys. Rev. A, 89,052714

Jonauskas, V., Kynienè, A., Merkelis, G., et al. 2015a, Phys. Rev. A, 91, 012715

Jonauskas, V., Pütterich, T., Kučas, S., et al. 2015b, J. Quant. Spectr. Rad. Transf., 160, 22

Karazija, R., \& Kučas, S. 2013, J. Quant. Spectr. Rad. Transf., 129, 131

Koncevičiūtė, J., Kučas, S., Masys, ک̌., Kynienė, A., \& Jonauskas, V. 2018, Phys. Rev. A, 97, 012705

Koncevičiūtė, J., Kučas, S., Kynienė, A., Masys, Š., \& Jonauskas, V. 2019, J. Phys. B: At. Mol. Opt. Phys., 52, 025203

Kučas, S., Jonauskas, V., \& Karazija, R. 1997, Phys. Scr, 55, 667

Kramida, A., Ralchenko, Y., \& Reader, J. 2018, NIST ASD Team 2018, NIST Atomic Spectra Database (ver. 5.5.6), available: https://physics.nist. gov, (Gaithersburg, MD: National Institute of Standards and Technology)

Kwon, D.-H., \& Savin, D. W. 2012, Phys. Rev. A, 86, 022701
Kynienè, A., Jonauskas, V., Kučas, S., \& Karazija, R. 2008, Lith. J. Phys., 48, 219

Kynienè, A., Masys, Š., \& Jonauskas, V. 2015, Phys. Rev. A, 91, 062707

Kynienè, A., Pakalka, S., Masys, Š., \& Jonauskas, V. 2016, J. Phys. B: At. Mol. Opt. Phys., 49, 185001

Landi, E., Young, P. R., Dere, K. P., Zanna, G. D., \& Mason, H. E. 2013, ApJ, 763,86

Linkemann, J., Müller, A., Kenntner, J., et al. 1995, Phys. Rev. Lett., 74, 4173

Loch, S., Ludlow, J., Pindzola, M., Whiteford, A., \& Griffin, D. 2005, Phys. Rev. A, 72,052716

Lotz, W. 1968, Z. Phys., 216, 241

Montague, R. G., Diserens, M. J., \& Harrison, M. F. A. 1984, J. Phys. B: At. Mol. Opt. Phys., 17, 2085

Mueller, D. W., Morgan, T. J., Dunn, G. H., Gregory, D. C., \& Crandall, D. H. 1985, Phys. Rev. A, 31, 2905

Palaudoux, J., Lablanquie, P., Andric, L., et al. 2010, Phys. Rev. A, 82, 043419 Pindzola, M. S., Griffin, D. C., \& Bottcher, C. 1986, Phys. Rev. A, 34, 3668 Pindzola, M. S., Griffin, D. C., Bottcher, C., Younger, S. M., \& Hunter, H. T. 1987, Nucl. Fusion, 27, 21

Pindzola, M. S., \& Loch, S. D. 2018, J. Phys. B: At. Mol. Opt. Phys., 51, 015202

Phillips, K. J. H., Feldman, U., \& Landi, E. 2012, Ultraviolet and X-ray Spectroscopy of the Solar Atmosphere (Cambridge: Cambridge Univ. Press)

Radžiūtė, L., Gaigalas, G., Kato, D., et al. 2015, J. Quant. Spectr. Rad. Transf., 152, 94

Stenke, M., Aichele, K., Hartenfeller, U., et al. 1999, J. Phys. B: At. Mol. Opt. Phys., 32, 3627

Wong, K. L., Beiersdorfer, P., Chen, M. H., \& Marrs, R. E. 1993, Phys. Rev. A, 48,2850

Zhang, D.-H., \& Kwon, D.-H. 2014, J. Phys. B: At. Mol. Opt. Phys., 47, 075202 